This item was submitted to Loughborough's Research Repository by the author.

Items in Figshare are protected by copyright, with all rights reserved, unless otherwise indicated.

\title{
Generation of ground elastic waves by road vehicles
}

PLEASE CITE THE PUBLISHED VERSION

http://dx.doi.org/10.1142/S0218396X01000632

PUBLISHER

World Scientific Publishing (๑ IMACS)

VERSION

AM (Accepted Manuscript)

LICENCE

CC BY-NC-ND 4.0

REPOSITORY RECORD

Krylov, Victor V.. 2012. "Generation of Ground Elastic Waves by Road Vehicles". figshare. https://hdl.handle.net/2134/9979. 
This item was submitted to Loughborough's Institutional Repository (https://dspace.lboro.ac.uk/) by the author and is made available under the following Creative Commons Licence conditions.

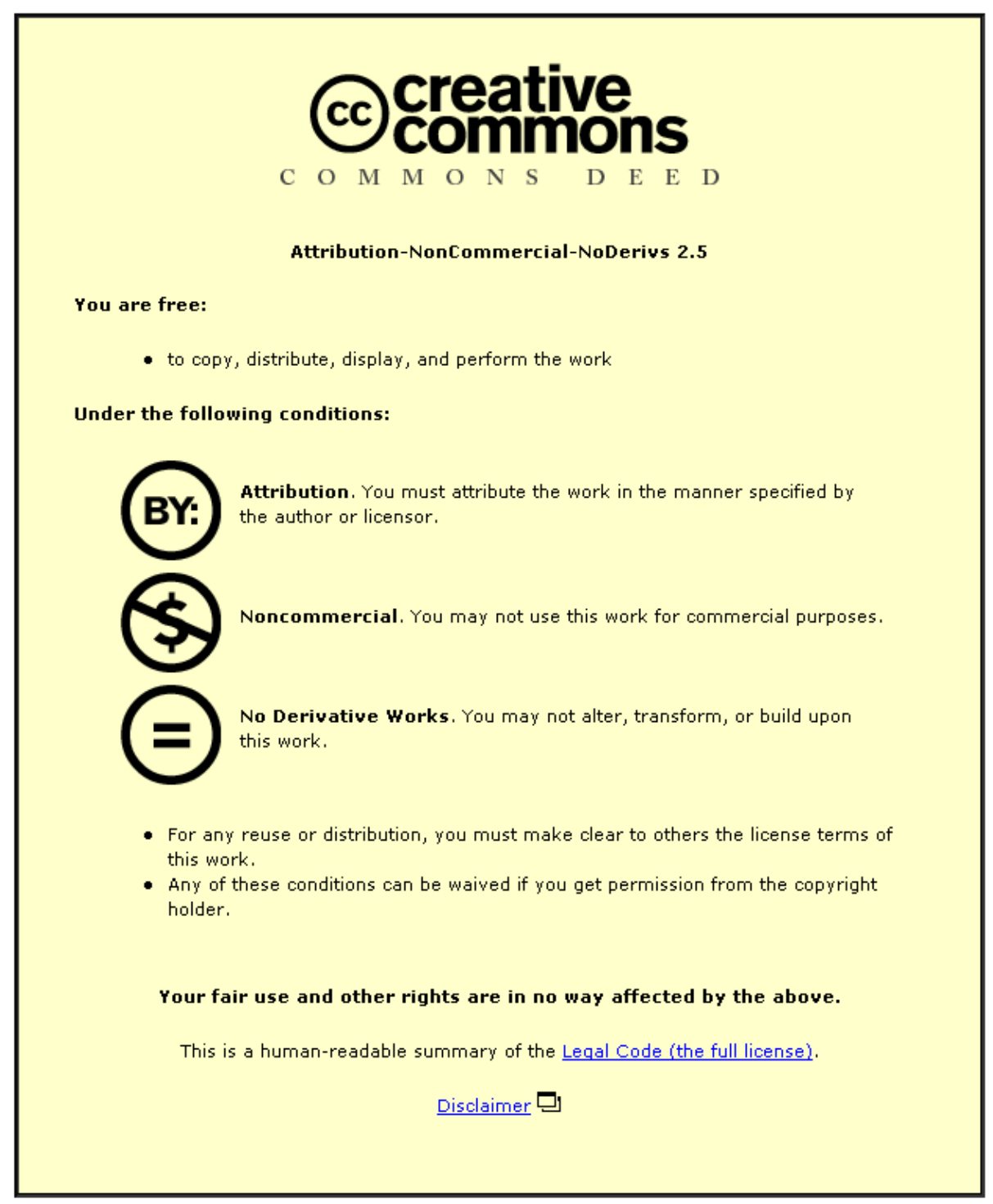

For the full text of this licence, please go to: http://creativecommons.org/licenses/by-nc-nd/2.5/ 
Journal of Computational Acoustics, Vol. 9, No. 3 (2001) 919-933

\title{
GENERATION OF GROUND ELASTIC WAVES BY ROAD VEHICLES
}

\author{
Victor V. Krylov \\ Department of Civil and Structural Engineering, \\ The Nottingham Trent University, \\ Burton Street, Nottingham NG1 4BU, UK
}

\begin{abstract}
Ground elastic waves, or ground-borne vibrations, represent one of the major adverse environmental impacts of road vehicles, especially of heavy lorries. In the present paper, ground elastic waves generated by road vehicles are investigated theoretically. Two main generation mechanisms are considered. The first one is associated with vehicles travelling on rough or bumpy road surfaces, in particular over road humps and speed cushions installed by local authorities at some sensitive road locations as a simple method of traffic calming. The second mechanism of generation is associated with acceleration and braking of road vehicles. General analytical results are illustrated by numerical examples and are compared with the existing experiments.
\end{abstract}

\section{INTRODUCTION}

Ground-borne vibrations represent one of the major adverse environmental impacts of road vehicles, especially of heavy lorries. Generally, one can distinguish two main mechanisms of generating ground vibrations by road vehicles (Watts 1984).

The first one is associated with vehicles travelling on rough or bumpy road surfaces, in particular over road humps and speed cushions installed by local authorities at some sensitive road locations as a simple method of traffic calming. As the practice show, installation of road humps and speed cushions results in significant reduction of the severity and number of road accidents as a result of decrease in speeds at which drivers choose to travel. However, the recent experimental investigations carried out by the UK Transport Research Laboratory (TRL) have demonstrated that such a gain is achieved partly in expense of the increased traffic noise and ground-borne vibration (Watts 1998). It has been shown during these investigations that speed control cushions and road humps can produce perceptible levels of ground vibration which depend on vehicle type and hump and cushion design.

The second mechanism of generation is associated with acceleration and braking of road vehicles. It is essential in the places where vehicles stop, start or change direction of their movement, e.g., before traffic lights or in supermarket yards.

Theoretical investigations of ground vibrations generated by road traffic have been carried out by several authors. A number of works has been done for vehicles travelling on statistically rough surfaces of rather good quality (Taniguchi et al. 1979, Le Houedec et al. 1982, Hunt 1991, Hanazato et al. 1991) and for accelerating and braking vehicles (Krylov 1995a-c, 1996a,b). However, vibrations caused by vehicles travelling over single obstacles 
were analysed only very briefly (Krylov 1995a,c) and no calculations directly relevant to traffic calming road humps and speed cushions were carried out. In the present paper, we describe a more comprehensive theory of ground elastic waves generated by road vehicles.

In the case of ground vibrations generated on bumpy roads, a vehicle is modelled as a mechanical system having four degrees of freedom associated with its main low-frequency resonances. Solving the system of the corresponding dynamic equations, the elastic Lame equation describing ground motion, and taking into account the boundary conditions on the ground surface, which incorporate dynamic forces caused by the interaction of a moving vehicle with a hump, results in the analytical expressions for generated ground vibration spectra. It is shown that amplitudes and spectra of generated ground vibrations depend on shapes and sizes of the road humps, on vehicle speeds and loads, and on ground elastic properties. In particular, the dependence on vehicle speeds demonstrates the initial increase of amplitudes as speeds increase and then their monotonous decrease according to the inverse proportionality law.

The problem of generating ground vibrations during acceleration and braking is considered for vehicles accelerating (decelerating) with a constant acceleration from rest to a constant speed, or braking to a stop from a constant speed. According to the low-frequency approximation considered, an accelerating or braking vehicle is modelled as a horizontal traction force proportional to its mass and acceleration and moving along with the vehicle. Frequency spectra of the generated surface waves are described as functions of acceleration and final (initial) speed of the vehicle. In particular, it is shown that for medium and upper bands of the spectra the generated ground vibration level is determined mainly by acceleration and is almost independent of the initial (final) vehicle speed. For low-frequency spectral bands, oscillations of ground vibration amplitudes versus both acceleration and initial speed may take place. These oscillations may be responsible for large statistical deviations of experimentally observed ground vibration levels.

\section{GROUND VIBRATIONS CAUSED BY ROAD HUMPS AND CUSHIONS}

\section{$\underline{2.1}$ Vehicle interaction with road humps}

In what follows we give a very brief description of the mechanism of vehicle interaction with road humps and speed cushions (Fig. 1) necessary for understanding the process of generating ground vibrations. It is assumed that both wheels of the front axle of a vehicle hit humps or cushions simultaneously. This process is then repeated for the rear wheel axle. Therefore, for modelling purposes both humps and cushions can be considered as twodimensional bumps extended to infinity in y-direction (which is not shown in Fig.1). As will be shown below, despite the two-dimensional bump geometry, the problem of wave generation by vehicle interacting with such bumps is three-dimensional. At low frequencies, typical for vehicle-generated ground vibrations, the resulting action of two wheels interacting with the bump can be modelled as a vertical point force applied to the ground surface at $x=$ $0, y=0$.

Typical mechanical model of a road vehicle travelling on uneven roads possesses four degrees of freedom corresponding to four main resonance frequencies of low-frequency vibrations related to body bounce and pitch, or to front- and rear-axle hops (Hunt 1991, 
Wong 1993). Frequencies of body bounce and pitch resonances are normally very low (in the range of 1-3 Hz). Axle-hop resonance frequencies are essentially larger (from $8 \mathrm{~Hz}$ to $12 \mathrm{~Hz}$ ) and are therefore more important from the point of view of generating ground vibrations (we remind the reader that ground wave generation efficiency is higher at higher frequencies).

Keeping this in mind, we use the simplified model of a vehicle, considering its carriage as immobile in vertical direction and taking into account only axle vibrations. This model consists of two identical vibrating systems, each having one degree of freedom and comprising an axle mass $m$ and two springs with constants $K_{1}$ and $K_{2}$ modelling respectively the elasticity of tyre and suspension (Fig. 2). Axles are separated from each other by the distance $L$ (wheel base). We also assume that a hump or cushion cross-section in the plane $y=0$ is described by the function $z_{1}=f(x)$ (see Fig. 1).

According to the model considered, the equation describing vertical displacements of each axle versus its static position $z_{2}$ has the form

$$
m \partial^{2} z_{2} / \partial^{2}+Q \partial z_{2} / \partial+K z_{2}=K_{1} z_{1}(v t)
$$

where $K=K_{1}+K_{2}$ is a combined elasticity of tyre and suspension, and $Q$ is a total damping coefficient.

Assuming that hump area is small as compared with wavelengths of generated vibrations and its centre is located at $x=0$ and $y=0$, the related normal stress $T_{z z}$ applied to the ground can be written in the form

$$
T_{z z}(\rho, t)=K_{1}\left[\left(z_{2}(t)-Z_{1}(t)+z_{2}(t-L / v)-Z_{1}(t-L / v)\right] \delta(x) \delta(y)\right.
$$

where $Z_{1}(t)=z_{1}(v t)$ and $Z_{1}(t-L / v)=z_{1}(v t-L)$ are the input functions for the front and rear axles respectively, $\rho=\{x, y\}$ is the surface radius-vector, and $\delta(z)$ is Dirac's delta-function.

Solving eqn (2.1) by Fourier method, one can derive the following expression for the Fourier transform $z_{2}(\omega)$ of a front wheel axle vertical displacement $z_{2}(t)$ :

$$
z_{2}(\omega)=\frac{\omega_{1}^{2} Z_{1}(\omega)}{\sqrt{\left(\omega_{0}^{2}-\omega^{2}\right)^{2}+(2 \omega \alpha)^{2}}} \exp \left[-i \tan ^{-1}\left(\frac{2 \omega \alpha}{\omega_{0}^{2}-\omega^{2}}\right)\right]
$$

where $\omega_{0}=(K / m)^{1 / 2}$ is the hop resonance frequency, $\omega_{1}=\left(K_{1} / m\right)^{1 / 2}$ is the tyre "jumping" resonant frequency, $\quad \alpha=Q / 2 m$ is a normalised damping coefficient, and $Z_{1}(\omega)$ is the Fourier spectrum corresponding to the hump profile. The Fourier transform for a rear wheel axle vertical displacement $z_{2}(t-L / v)$ differs from (2.3) only in phase shift $\omega L / v$ and is omitted here for shortness. The Fourier transform of the vertical force applied from the vehicle to the ground, $T_{z z}(\rho, \omega)$, is easily obtained from (2.2) via replacing $z_{2}(t), Z_{1}(t), z_{2}(t-$ $L / v)$, and $\left.Z_{1}(t-L / v)\right]$ by their Fourier spectra:

$$
T_{z z}(\rho, \omega)=K_{1}\left[\left(z_{2}(\omega)-Z_{1}(\omega)+z_{2}(\omega) e^{i \omega L / v}-Z_{1}(\omega) e^{i \omega L / v}\right] \delta(x) \delta(y) .\right.
$$




\subsection{Calculation of generated elastic waves}

The ground vibration field generated by vehicles in an elastic half space, which we assume to be homogeneous and isotropic, should satisfy the elastic Lame' equation

$$
(\lambda+2 \mu) \operatorname{grad} \operatorname{div} \boldsymbol{u}-\mu \operatorname{rot} \operatorname{rot} \boldsymbol{u}-\rho_{0} \partial^{2} \mathbf{u} / \partial^{2}=0,
$$

and the boundary conditions on the ground surface taking into account the vertical force (2.2) resulting from the interaction of a vehicle with an uneven surface. Here $\boldsymbol{u}$ is the particle displacement vector with the components $u_{i} ; \lambda$ and $\mu$ are the elastic Lame' constants; and $\rho_{0}$ is the ground mass density.

The ground surface geometry is rather complex due to the presence of road humps and cushions. However, since the characteristic height $h$ of road humps and cushions is very small in comparison with characteristic wavelengths $\lambda_{v}$ of generated vibrations, it can be simplified by using the method of projection of the actual boundary conditions onto the flat surface $z=0$. One can show that the projected boundary conditions differ from the real ones only by the additional small terms proportional to $h / \lambda_{v}<<1$. In further consideration we will neglect these terms and write the boundary conditions at $z=0$ in the form:

$$
\begin{aligned}
& \sigma_{x z}=2 \mu u_{x z}=0, \\
& \sigma_{y z}=2 \mu u_{y z}=0, \\
& \sigma_{z z}=\lambda u_{n n}+2 \mu u_{z z}=-T_{z z}(\rho, t) .
\end{aligned}
$$

Here $u_{i j}=(1 / 2)\left(\partial u_{i} / \partial x_{j}+\partial u_{j} / \partial x_{i}\right)$, where $i, j=1,2,3$, are the components of the linearised deformation tensor; and $\sigma_{i j}$ are the components of the elastic stress tensor. Without loss of generality, we limit our calculation of frequency spectra to the vertical component of ground vibration velocity $v_{z}=d u_{z} / d t$ which is usually measured during experimental observations.

Solving the boundary value problem (2.2)-(2.6) by the Green's function method (see, e.g., Ewing et al. 1957; Graff 1975) and taking into account only generated Rayleigh waves, one can derive the following expression for the vertical component of the surface vibration velocity spectrum:

$$
v_{\mathrm{z}}(\rho, \omega)=\left(\frac{2 \pi}{k_{R} \rho}\right)^{1 / 2} \frac{(-i \omega) k_{R} k_{t}^{2} v_{l}}{2 \pi \mu F^{\prime}\left(k_{R}\right)} T_{z z}(\omega) e^{-k_{R} \gamma \rho} e^{i k_{R} \rho-i 3 \pi / 4}
$$

Here $T_{z z}(\omega)=T_{z z}(0, \omega)$ (see eqn (2.4)); $\rho=\rho(x, y)$ is the distance to the observation point; $k_{R}=\omega / c_{R}$ is the Rayleigh wave number, where $c_{R}$ is Rayleigh wave velocity; $v_{l, t}=\left(k_{R}^{2}\right.$ $\left.k_{l, t}^{2}\right)^{1 / 2}$ are nonspecified expressions, where $k_{l, t}=\omega / c_{l, t}$ are the wavenumbers of bulk longitudinal and shear acoustic waves, $c_{l}$ and $c_{t}$ are their phase velocities; $F^{\prime}\left(k_{R}\right)$ is the derivative $d F(k) / d k$ of the Rayleigh determinant $F(k)=\left(2 k^{2}-k_{t}^{2}\right)^{2}-4 k^{2} v_{l} v_{t}$ taken at $k=k_{R}$.

In writing (2.7) we have taken account of attenuation of generated ground vibrations in the ground by replacing the wavenumber of a Rayleigh wave in an ideal elastic medium $k_{R}=$ $\omega / c_{R}$ by the complex wavenumber $k_{R}{ }^{\prime}=k_{R}(1+i \gamma)=\left(\omega / c_{R}\right)(1+i \gamma)$. Here $\gamma<<1$ is a positive 
constant which describes the linear dependence of a Rayleigh wave attenuation coefficient on frequency $\omega$. For different types of ground $\gamma$ are in the range from 0.01 to 0.2 (Gutovski et al. 1976). In what follows we will be interested only in amplitudes of ground vibrations $V_{z}(\rho, \omega)=\left|v_{z}(\rho, \omega)\right|$, ignoring the phase information.

\section{$\underline{2.3 \text { Numerical calculations and discussion }}$}

Numerical calculations of ground vibrations generated by vehicles crossing humps and cushions have been carried out according to eqn (2.7) for vehicles travelling at different speeds over road humps and cushions of different profiles and dimensions. Vehicle parameters were: $\omega_{1}=65 \mathrm{rad} / \mathrm{s}, \omega_{0}=70 \mathrm{rad} / \mathrm{s}, \alpha=15, L=6 \mathrm{~m}$, and $\mathrm{m}=50 \mathrm{~kg}$. Ground parameters were: Rayleigh wave velocity $c_{R}=125 \mathrm{~m} / \mathrm{s}$, Poison ratio $\sigma=0.25$, ground mass density $\rho_{0}=2000 \mathrm{~kg} / \mathrm{m}^{3}$, ground attenuation constant $\gamma=0.05$. Observation distance was $\rho=30 \mathrm{~m}$.

Obviously, if the spectrum $Z_{1}(\omega)$ has components around the axle hop resonance frequency $\omega_{0}$, then the axle vibrations are effectively excited (Fig. 3) and noticeable generation of ground vibrations takes place (see Fig. 4). Analysis and numerical calculations show that amplitudes and spectra of generated ground vibrations depend on hump and cushion profile, their characteristic gradient (ratio $h / l$ ), vehicle speed $v$ and load, and elastic parameters of the ground.

In particular, the smoother the hump the lower the level of generated ground vibrations. For example, for typical vehicle speeds $v$, a bell-shaped hump, $z_{1}(x)=h \exp \left(-x^{2} / l^{2}\right)$, causes approximately $20 \mathrm{~dB}$ reduction in the integral level of generated ground vibrations in comparison with a cosine-shaped hump, $z_{1}(x)=h \cos (\pi x / l)$, characterised by the same values of $h$ and $l$.

Calculations for cosine-shaped road humps, including the ones with typical parameters: $h$ $=0.074 \mathrm{~m}, l=0.9 \mathrm{~m}$; and $h=0.064 \mathrm{~m}, l=3.7 \mathrm{~m}$, have demonstrated that the integral level of generated ground vibrations grows roughly in direct proportion to $h / l$. This agrees well with the experimental results of TRL for two round-shaped profiles of the above mentioned dimensions (Watts 1998).

Dependence of generated ground vibrations on vehicle speed $v$ is quite complex for each particular spectral component. Calculated results for a cosine-shaped hump are shown in Fig. 5. One can see that for small and medium speeds $v$ ground vibration amplitudes grow with oscillations (depending on hump length $l$ and wheel base $L$ ) and reach a maximum at certain $v$. Then, for larger values of $v$, the level of generated vibrations decays inversely proportionally to $v$. Calculation of the integral level of generated ground vibrations carried out for the same cosine-shaped hump shows that it depends on $v$ in a more simple way (Fig. 6). The dependence is roughly linear for typical values of $v$ at which drivers chose to travel in traffic calming areas (from 0 to $7 \mathrm{~m} / \mathrm{s}$ ). Again, the initial average growth of generated ground vibrations with the increase of vehicle speed agrees with the TRL experimental results (Watts 1998), although no detailed comparison is possible because of the insufficient number of vehicle speeds (four) used in the experimental observations.

Note that heavy loads result in the increase of static stresses applied to the vehicle suspension which, due to nonlinearity of material stress-strain relations, will change the stiffness of an equivalent spring and, hence, modify the axle hop resonance frequency $\omega_{0}$. The directions of these modifications are different for steel and air suspensions: for the first 
case the frequency $\omega_{0}$ decreases, whereas for the latter case it increases. According to the above theory and in agreement with the experiments (Watts 1998), these may result in a small decrease in generated vibrations for vehicles with steel suspension and in their small increase for vehicles with air suspension.

The presence of the ground shear modulus $\mu$ in the denominator of eqn (2.7) implies that for the softer ground (smaller values of $\mu$ ) the generated ground vibrations are more intensive.

\section{GROUND VIBRATIONS CAUSED BY ACCELERATION AND BRAKING}

\subsection{Traction forces applied to the ground}

In this section we consider generation of ground vibrations by vehicles accelerating at constant acceleration $a$ from rest to a constant speed $v$, or braking with constant acceleration $-a$ from $v$ to a complete stop. The assumption of constant acceleration (deceleration) is in agreement with general requirements for vehicle performance, e.g., in the process of braking. According to these requirements (Newcomb et al 1967), deceleration produced during braking should ideally be uniform throughout an application.

It should be emphasised that, in contrast to the normal load forces considered in the previous section, the main mechanism of ground vibration generation by accelerating or decelerating vehicles is the action of horizontal traction forces which are applied from tyres to the ground only during the time of accelerating or braking (the contribution of normal load forces is relatively small for speeds and accelerations typical for road traffic). Note that the horizontal interaction forces, acting during deceleration between vehicle and road through the tyre-road interface, are limited by friction (adhesion) between tyre and road. If the product of deceleration and vehicle mass exceeds this friction, sliding or skidding will occur.

Road braking statistics show that in the majority of cases decelerations range from 0.1 to $0.2 \mathrm{~g}$, i.e. from about 1 to $2 \mathrm{~m} / \mathrm{s}^{2}$. However, in emergency braking decelerations of $0.4-0.6 \mathrm{~g}$ are typical, and greater than $1.0 \mathrm{~g}$ is possible. In what follows we consider vehicle acceleration $\pm a$ as a given value resulting from joint application of engine or braking forces, drag forces caused by wind resistance, rolling resistance, friction in the wheel bearings etc (Newcomb et al 1967; Wong 1993). We also assume that the most important internal degrees of freedom of the vehicle (corresponding to the pitch, bounce and wheel resonances are not excited by horizontal inertial forces produced during acceleration or deceleration. Being interested only in the low-frequency range of generated ground vibrations, we will model an accelerating or breaking vehicle of mass $M$ as a point horizontal traction force $F_{X}=a M$ applied to the ground and moving along the road at vehicle speed. In fact, the resulting point force is the sum of partial forces applied to the ground from each wheel of the vehicle. For braking or accelerating respectively the direction of this force coincides with the direction of vehicle movement or is opposite to it. We also assume that adhesion between road and tyres is perfect. Thus, the limitations on acceleration (deceleration) caused by sliding or skidding will not be considered. 


\subsection{Governing equations and their formal solution}

If a vehicle is accelerated or decelerated at constant absolute value $a$, then the point load force applied from a vehicle to the ground surface is a horizontal traction force that moves along the $x$-axis with a vehicle (see Fig. 7) and has the amplitude

$$
F_{X}=a M
$$

The related mechanical shear stresses applied to the ground surface during acceleration or deceleration, i.e. during the period of time from $t=0$ to $t=v / a$, where $v$ is the final or initial speed, are described respectively as follows:

and

$$
T_{x z}(\rho, t)=-F_{x} \delta\left(x-a t^{2} / 2\right) \delta(y),
$$

$$
T_{x z}(\rho, t)=F_{x} \delta\left(x-v t+a t^{2} / 2\right) \delta(y) .
$$

Here $T_{x z}$, are the components of a load stress tensor applied to the surface, $\rho=\{x, y\}$ is the surface radius-vector, and $\delta(z)$ is Dirac's delta-function.

The ground vibration field generated by accelerating or braking vehicles in an elastic half space should satisfy the earlier written elastic Lame' equation (2.5) and the boundary conditions on the ground surface which now take into account horizontal traction forces (3.2) and (3.3):

$$
\begin{aligned}
& \sigma_{x z}=2 \mu u_{x z}=-T_{x z}(\rho, t), \\
& \sigma_{y z}=2 \mu u_{y z}=0, \\
& \sigma_{z z}=\lambda u_{n n}+2 \mu u_{z z}=0 .
\end{aligned}
$$

The solution to the above problem can be written as the following Fourier integral:

$$
u_{i}(\boldsymbol{r}, t)=\left(1 / 4 \pi^{2}\right) \int_{-\infty}^{\infty} \int_{-\infty}^{\infty} \int_{-\infty}^{\infty} T_{x z}(\omega, \boldsymbol{k}) G_{x i}(\omega, \boldsymbol{k}, z) e^{-i \omega t+\boldsymbol{i}(\boldsymbol{k} \rho)} d \omega d \boldsymbol{k},
$$

where $\boldsymbol{r}=\{x, y, z\}$ is the three-dimensional radius-vector of the observation point, $\rho(x, y)=$ $\{x, y, 0\}$ is the projection of the vector $\boldsymbol{r}$ onto the plane $z=0, \boldsymbol{k}=\left\{k_{x}, k_{y}\right\}$ is the projection of a current three-dimensional wave-vector onto the plane $z=0$, and $\omega$ is the circular frequency. Functions $T_{x z}(\omega, \boldsymbol{k})$ and $G_{x i}(\omega, \boldsymbol{k}, z)$ are respectively the Fourier transforms of the corresponding components of a load force source and the dynamic Green's tensor (Green's function) which are related to the space-time representations by the following formulae:

$$
T_{x z}(\omega, \boldsymbol{k})=(1 / 2 \pi) \int_{0}^{\mathrm{v} / \mathrm{a}} \int_{-\infty-\infty}^{\infty} \int_{x z}^{\infty} T_{x,-\infty}(x) e^{i \omega t-i(\boldsymbol{k} \rho)} d \rho d t
$$

and 


$$
G_{x i}(\omega, \boldsymbol{k}, z)=(1 / 2 \pi) \int_{-\infty}^{\infty} \int_{-\infty-\infty}^{\infty} \int_{-\infty}^{\infty} G_{x i}(\rho, t, z) e^{i \omega t-i(\boldsymbol{k} \rho)} d \rho d t
$$

The Fourier transform of the relevant component $G_{x z}(\omega, \boldsymbol{k}, z)$ of the dynamic Green's tensor for an elastic half space is:

$$
G_{x z}(\omega, \boldsymbol{k}, z)=i \frac{2 k_{x} v_{l} v_{t}}{\mu F(k)} e^{-v_{l} z}+i \frac{k_{x}}{\mu F(k) k_{t}^{2}}\left[F(k)-2 k^{2} b(k)\right] e^{-v_{t} z} .
$$

Here the following notation is used: $k=\left(k_{x}^{2}+k_{y}^{2}\right)^{1 / 2}, b(k)=2 k^{2}-k_{t}^{2}-2 v_{l} v_{t}, \quad v_{l, t}=\left(k^{2}-\right.$ $\left.k_{l, t}^{2}\right)^{1 / 2} ; k_{l, t}=\omega / c_{l, t}$ are the wavenumbers of bulk longitudinal and shear acoustic waves; $c_{l}=$ $\left[(\lambda+2 \mu) / \rho_{0}\right]^{1 / 2}$ and $c_{t}=\left(\mu / \rho_{0}\right)^{1 / 2}$ are their phase velocities; $F(k)=\left(2 k^{2}-k_{t}^{2}\right)^{2}-4 k^{2} v_{l} v_{t}$ is the Rayleigh determinant.

Using eqn (3.8) in eqn (3.5) and applying the equality $v_{z}(\boldsymbol{r}, \omega)=-i \omega u_{z}(\boldsymbol{r}, \omega)$, one can determine the spectrum of the vertical component of the ground vibration velocity which takes into account only Rayleigh waves:

$$
\begin{aligned}
v_{Z}(\mathbf{r}, \omega)= & \left(\frac{2 \pi}{k_{R} \rho}\right)^{1 / 2} \frac{\omega k_{R}^{2}\left[k_{t}^{2} v_{l} v_{t}-k_{R}^{2} b\left(k_{R}\right)\right] \cos \Theta}{\pi \mu F^{\prime}\left(k_{R}\right) k_{t}^{2}} \times \\
& T_{X Z}\left(\omega, k_{R} \cos \Theta\right) e^{-k_{R} \gamma \rho} e^{i k_{R} \rho-i 3 \pi / 4}
\end{aligned}
$$

where $\Theta=\cos ^{-1}(x / \rho)$ is the observation angle and $F^{\prime}\left(k_{R}\right)$ is the derivative $d F(k) / d k$ taken at $k=k_{R}$.

Let us now discuss the Fourier transforms $T_{x z}\left(\omega, k_{R} \cos \theta\right)$ of the load forces (eqns (3.2) and (3.3)). Substituting eqns (3.2) and (3.3) into (3.6) and performing integration over $x$ and $y$ with taking into account properties of the delta-function, one can obtain the following expressions for accelerating and braking vehicles respectively:

$$
\begin{aligned}
& T_{x z}\left(\omega, k_{R} \cos \theta\right)=-(1 / 2 \pi) a M \int_{0}^{\mathrm{v} / \mathrm{a}} \mathrm{e}^{\mathrm{i} \omega \mathrm{t}-\mathrm{ik}_{\mathrm{R}} \cos \Theta\left(\mathrm{at}^{2} / 2\right)} \mathrm{dt}, \\
& T_{X z}\left(\omega, k_{R} \cos \theta\right)=(1 / 2 \pi) a M \int_{0}^{\mathrm{v} / \mathrm{a}} \mathrm{e}^{\mathrm{i}\left(\omega-\mathrm{k}_{\mathrm{R}} \mathrm{v} \cos \Theta\right) \mathrm{t}+\mathrm{ik}_{\mathrm{R}} \cos \Theta\left(\mathrm{at}^{2} / 2\right)} \mathrm{dt} .
\end{aligned}
$$

Taking these integrals analytically gives very bulky expressions containing functions of the complex variable $\operatorname{erf}(\mathrm{z})$, which are inconvenient for numerical computations. The only useful conclusion derived from these expressions follows from their comparison. Such a comparison shows that they differ from each other only by the phase factor. Thus, if one is interested only in the amplitudes of generated vibrations, it is sufficient to consider just one of these cases, e.g. the case of braking vehicles. In what follows we will be interested mainly in $1 / 3$ octave spectra of generated ground vibrations. This also allows us to use the approximate 
calculations of the integrals (3.10) and (3.11) without losing accuracy of calculations (for more details see Krylov 1996a,b).

\subsection{Numerical calculations and discussion}

The behaviour of $1 / 3$-octave spectra of ground vibrations (in $\mathrm{dB}$ relative to the reference level of $10^{-9} \mathrm{~m} / \mathrm{s}$ ) generated by a braking lorry with $M=20000 \mathrm{~kg}$ for three different values of deceleration: $a=1,5$ and $9 \mathrm{~m} / \mathrm{s}^{2}$ is illustrated in Fig. 8. The initial speed is $v=10 \mathrm{~m} / \mathrm{s}$. Other parameters are the following: mass density of the ground $\rho_{0}$ is $2000 \mathrm{~kg} / \mathrm{m}^{3}$, velocity of longitudinal bulk waves $-c_{l}=471 \mathrm{~m} / \mathrm{s}$, shear waves $-c_{t}=272 \mathrm{~m} / \mathrm{s}$ and Rayleigh surface waves $-250 \mathrm{~m} / \mathrm{s}$ (this corresponds to a Poisson ratio of $\sigma=0.25$ ). The constant of ground attenuation of Rayleigh waves was set as $\gamma=0.05$. It follows from Fig. 8 that amplitudes of generated ground vibrations at all frequencies increase with increase of $a$.

The behaviour of generated ground vibration spectra for three different values of the initial speed ( $v=5,10$ and $20 \mathrm{~m} / \mathrm{s})$ is shown on Fig. 9 for $\mathrm{a}=5 \mathrm{~m} / \mathrm{s}^{2}$ and $\Theta=\pi / 3$. It is seen that for frequencies higher than $4-5 \mathrm{~Hz}$ the spectra are almost independent of $v$.

The behaviour of generated ground vibrations (in $\mathrm{m} / \mathrm{s}$ ) as functions of acceleration $a$ and initial vehicle speed $v$ is illustrated respectively on Fig. 10 and Fig. 11. The ground vibration velocities were averaged over $1 / 3$-octave band around central frequencies $F=4,8$, 16, 32 and $64 \mathrm{~Hz}$. Note that oscillations of ground vibration level versus $a$ take place for larger $a$, whereas oscillations versus $v$ occur for smaller $v$. Also, the ground vibration velocity $V$ is proportional to $a$ for higher frequencies and for lower accelerations (Fig. 10) and does not depend on $v$ for higher frequencies and higher $v$ (Fig. 11). It is interesting that, according to Fig. 11, for low-frequency components of ground vibration spectra (4 and $8 \mathrm{~Hz}$ ) the level of generated vibrations may differ by about $12 \mathrm{~dB}$ if initial speed $v$ changes from 1 to $2 \mathrm{~m} / \mathrm{s}$. Thus, even small changes of $v$ in this range may result in large difference in ground vibrations generated in infrasound frequency range. This may cause high statistical deviations in levels of ground vibration generated by slowly approaching vehicles.

\section{CONCLUSIONS}

Theoretical analysis of ground vibrations generated by vehicles travelling over traffic calming road humps and cushions shows that amplitudes and spectra of vibrations depend strongly on the shape of hump or cushion profile, relation between its height and length, vehicle speed and load, and ground elastic parameters. The developed theoretical model of generating ground vibrations agrees with the existing experiments and can be successfully used in practice.

Study of ground vibrations generated by accelerating and braking vehicles shows that for medium and upper bands of the spectra the generated ground vibration level is determined mainly by acceleration and is almost independent of the initial (final) vehicle speed. For low-frequency spectral bands, oscillations of ground vibration amplitudes versus both acceleration and initial speed may take place. These oscillations may be responsible for large statistical deviations of experimentally observed ground vibration levels. 


\section{REFERENCES}

Ewing, W.M., Jardetzky, W.S. and Press, F. (1957). Elastic waves in layered media. McGraw-Hill, New York, N.Y.

Graff, K.F. (1975). Wave motion in elastic solids. Clarendon Press, Oxford.

Gutovski T.G., Dym C.L. (1976). Propagation of ground vibration: a review. Journ. of Sound and Vibration, 49, 179-193.

Hanazato, T., Ugai, K., Mori, M. and Sakaguchi, R. (1991). Three-dimensional analysis of traffic-induced ground vibrations. Journal of Geotechnical Engineering, 117, 11331151.

Hunt, H.E.M. (1991). Stochastic modelling of traffic-induced ground vibrations. Journal of Sound and Vibration, 144, 53-70.

Krylov, V.V. (1995a). Comparison of two main mechanisms of generating ground vibrations by road vehicles. Proc. I.O.A., 17(4), 75-82.

Krylov, V.V. (1995b). Ground vibrations from accelerating and braking road vehicles. In: Proc. 15th International Congress on Acoustics, Trondheim, Norway, 26-30 June 1995, Ed. M. Newman, Vol. 2, p. 161-164.

Krylov, V.V. (1995c) Generation of low-frequency Rayleigh waves by heavy lorries. Journ. Low Frequency Noise and Vibr., 14(4), 165-172.

Krylov, V.V. (1996a). Computation of ground vibrations generated by accelerating and braking road vehicles. Journ. of Vibration and Control (USA), 2, 299-321.

Krylov, V.V. (1996b). Generation of ground vibrations by accelerating and braking road vehicles. Acustica-acta acustica, 82(4), 642-649.

Le Houedec, D. and Riou, Y. (1982). Analytic response of a semi-infinite soil mass to road traffic vibration, Proceedings of the Conference on Soil Dynamics and Eartquake Engineering, Southampton, pp. 209-233.

Newcomb, T.P. and Spurr, R.T. (1967). Braking of road vehicles. Chapman and Hall, London.

Taniguchi, E. and Sawada, K. (1979). Attenuation with distance of traffic-induced vibrations, Japanese Society of Soil Mechanics and Foundation Engineering: Soils and Foundations 19, 15-28.

Watts, G.R. (1984). Vibration nuisance from road traffic - results of a 50 site survey. Transport Research Laboratory report LR1119 (Crowthorne).

Watts, G.R. (1998). Vehicle generated ground-borne vibration alongside speed control cushions and road humps. In: Ground Dynamics and Man-made Processes: Prediction, Design, Measurement, Ed. B.O. Skipp, Thomas Telford, London 1998, p. 87-101.

Wong, J.Y. (1993). Theory of ground vehicles, John Wiley \& Sons, New York. 


\section{FIGURE CAPTIONS}

Fig. 1. Two-dimensional bump modelling typical road humps and cushions

Fig. 2. Simplified mechanical model of a vehicle taking into account only axle hope resonances: two front wheels and two rear wheels are modelled as single masses $m$ supported by combined tyre stiffness $K_{1}$ and suspension stiffness $K_{2}$

Fig. 3. Fourier spectra $Z_{1}(F)$ (curve $z 1$ ) and $z_{2}(F)$ (curve $z 2$ ) for a vehicle travelling at speed $v=10 \mathrm{~m} / \mathrm{s}$ over the cosine-shaped hump with the parameters $l=0.9 \mathrm{~m}$ and $h=0.074 \mathrm{~m}$

Fig. 4. Spectrum of ground vibrations generated by a vehicle travelling at speed $v=5 \mathrm{~m} / \mathrm{s}$ over the cosine-shaped hump with the parameters $l=0.9 \mathrm{~m}$ and $h=0.074 \mathrm{~m}$

Fig. 5. Ground vibration amplitude at frequency $\mathrm{F}=11 \mathrm{~Hz}$ as a function of vehicle speed. Parameters of the hump are the same as in Figs. 3 and 4

Fig. 6. Integral value of vehicle-generated ground vibrations as a function of vehicle speed. Parameters of the hump are the same as in Figs. 3, 4 and 5

Fig. 7. Traction force $F_{x}$ applied to the ground during vehicle braking

Fig. 8. 1/3-octave ground vibration spectra calculated for three values of vehicle acceleration (deceleration) $a: a=1 \mathrm{~m} / \mathrm{s}^{2}$ (curve $V 1$ ), $a=5 \mathrm{~m} / \mathrm{s}^{2}$ (curve $V 2$ ) and $a=9 \mathrm{~m} / \mathrm{s}^{2}$ (curve V3)

Fig. 9. 1/3-octave ground vibration spectra calculated for three values of final (initial) vehicle speed $v: v=5 \mathrm{~m} / \mathrm{s}$ (curve $V 1$ ), $v=10 \mathrm{~m} / \mathrm{s}$ (curve $V 2$ ) and $v=20 \mathrm{~m} / \mathrm{s}$ (curve V3)

Fig. 10. Ground vibration amplitudes (in $\mathrm{m} / \mathrm{s}$ ) averaged over 1/3-octave frequency band as functions of vehicle acceleration (deceleration) $a$ for the central frequencies $F=$ 4,8,16, 32 and $64 \mathrm{~Hz}$ - curves V1, V2, V3, V4 and V5 respectively

Fig. 11. Ground vibration amplitudes (in $\mathrm{m} / \mathrm{s}$ ) averaged over 1/3-octave frequency band as functions of final (initial) vehicle speed $v$ for the central frequencies $F=4,8,16$, 32 and $64 \mathrm{~Hz}$ - curves $V 1, V 2, V 3, V 4$ and V5 respectively 


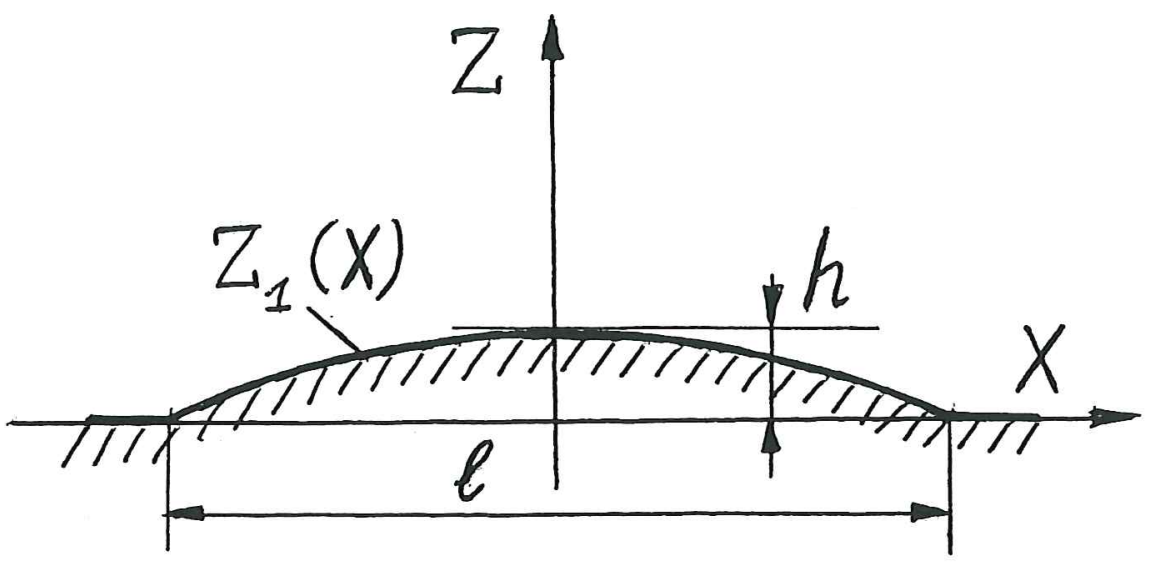

Fig. 1

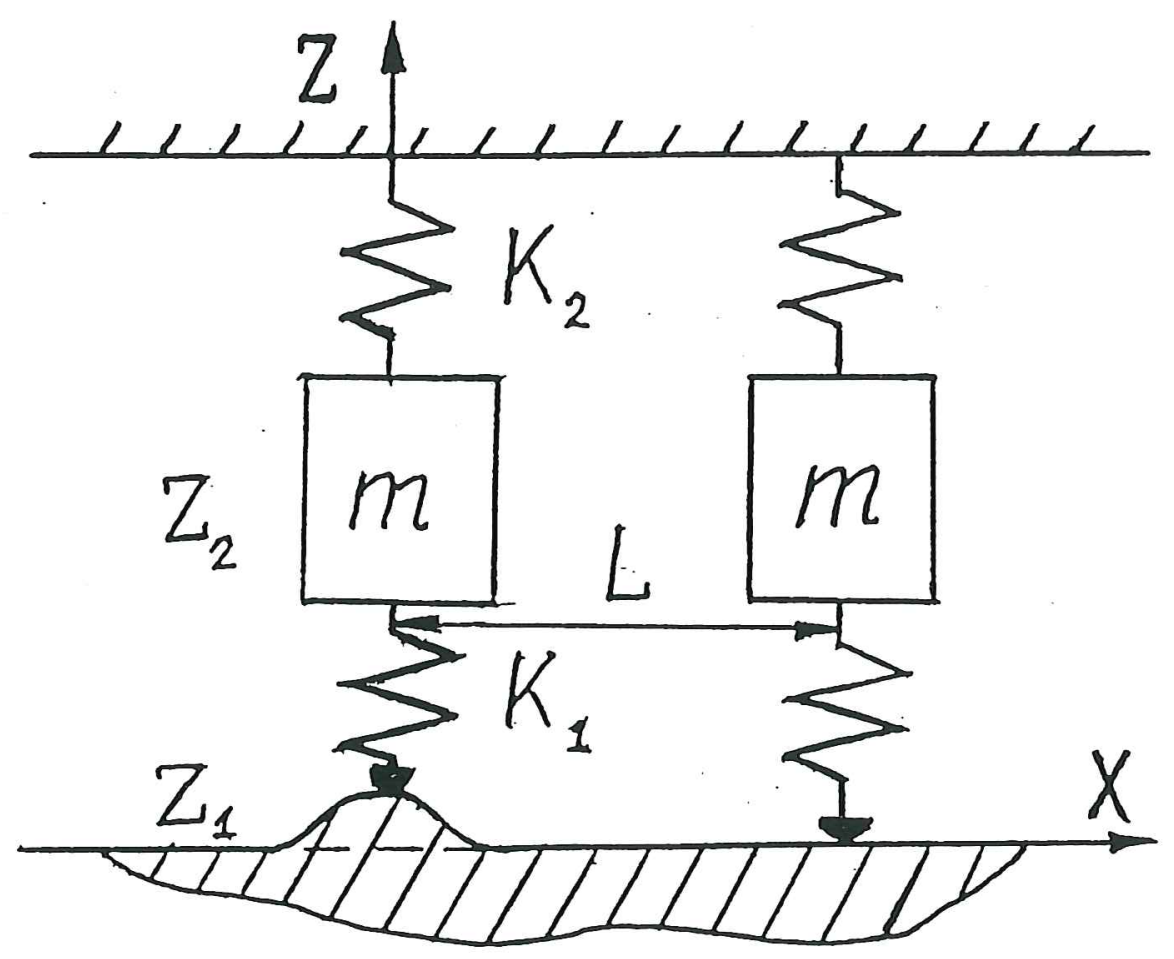

Fig. 2 


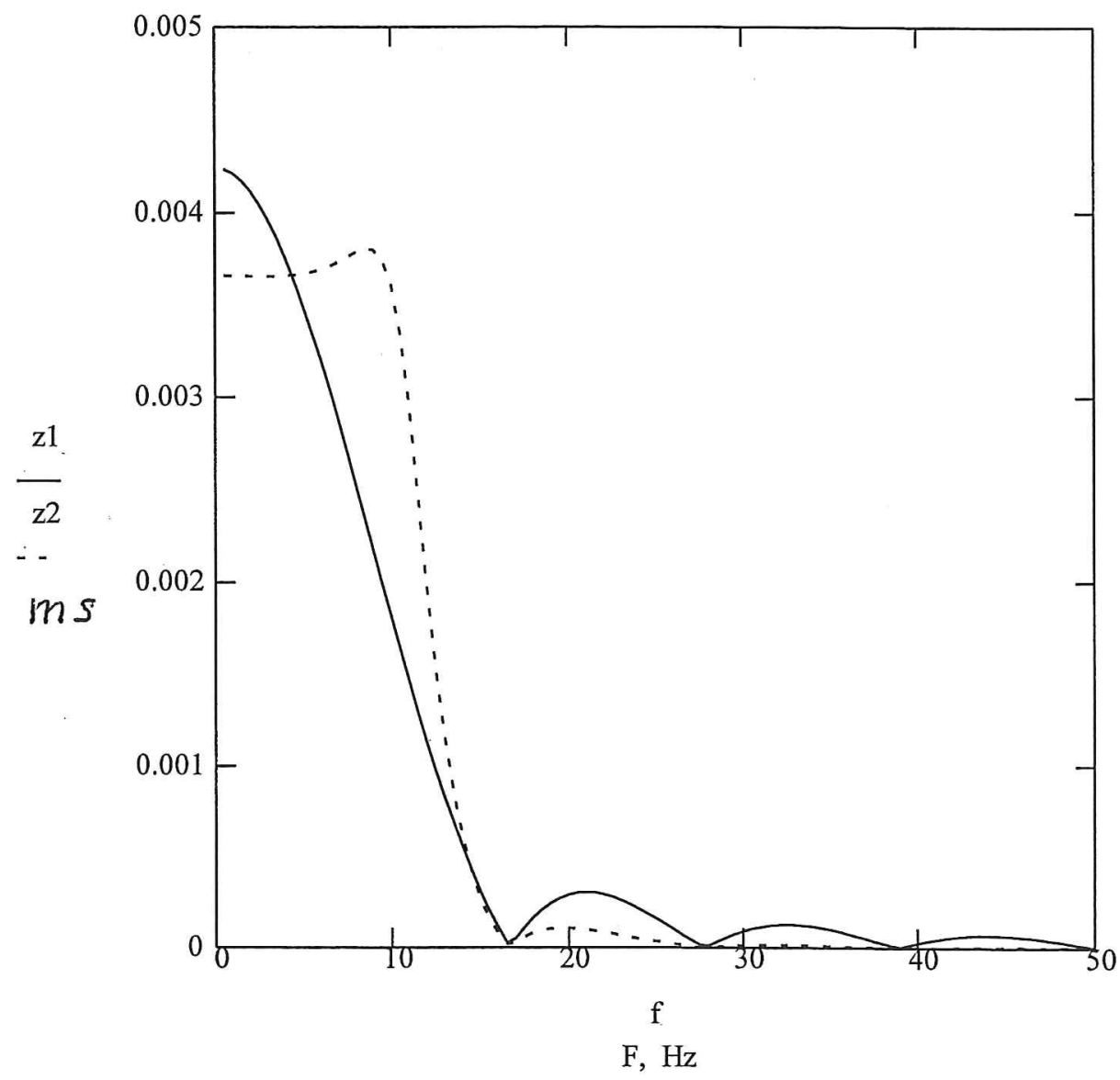

Fig. 3 


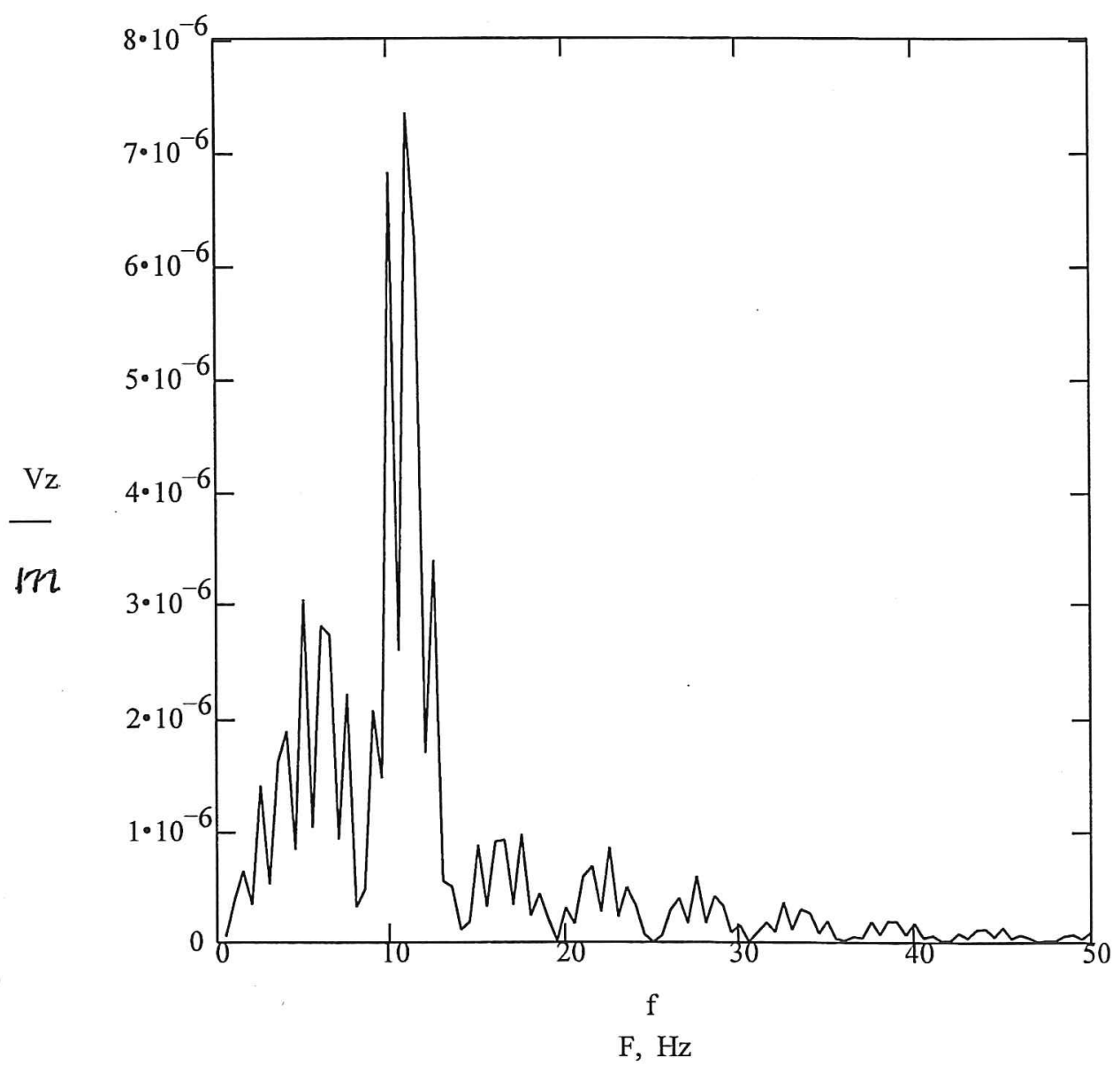

Fig. 4 


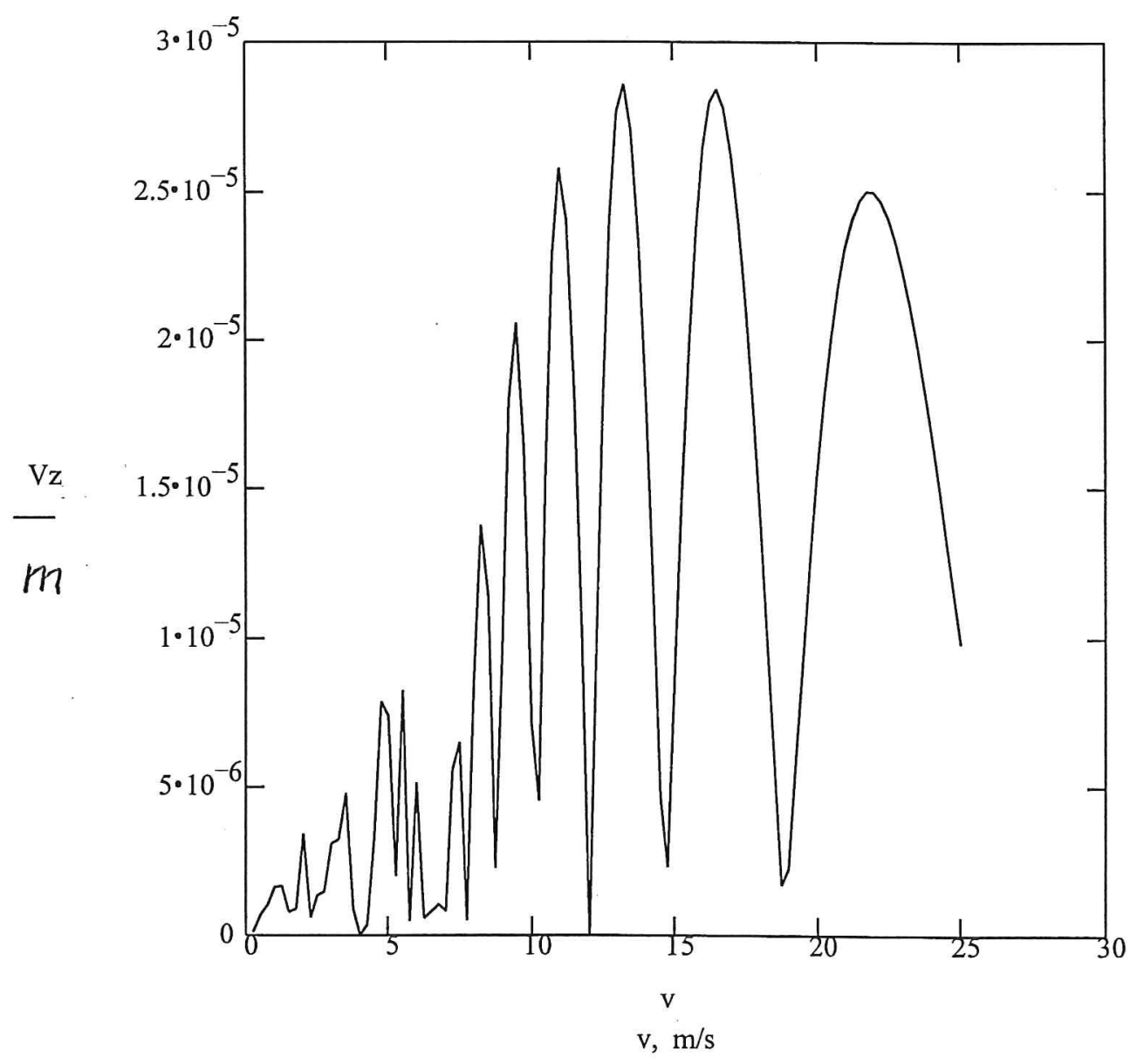

Fig. 5 


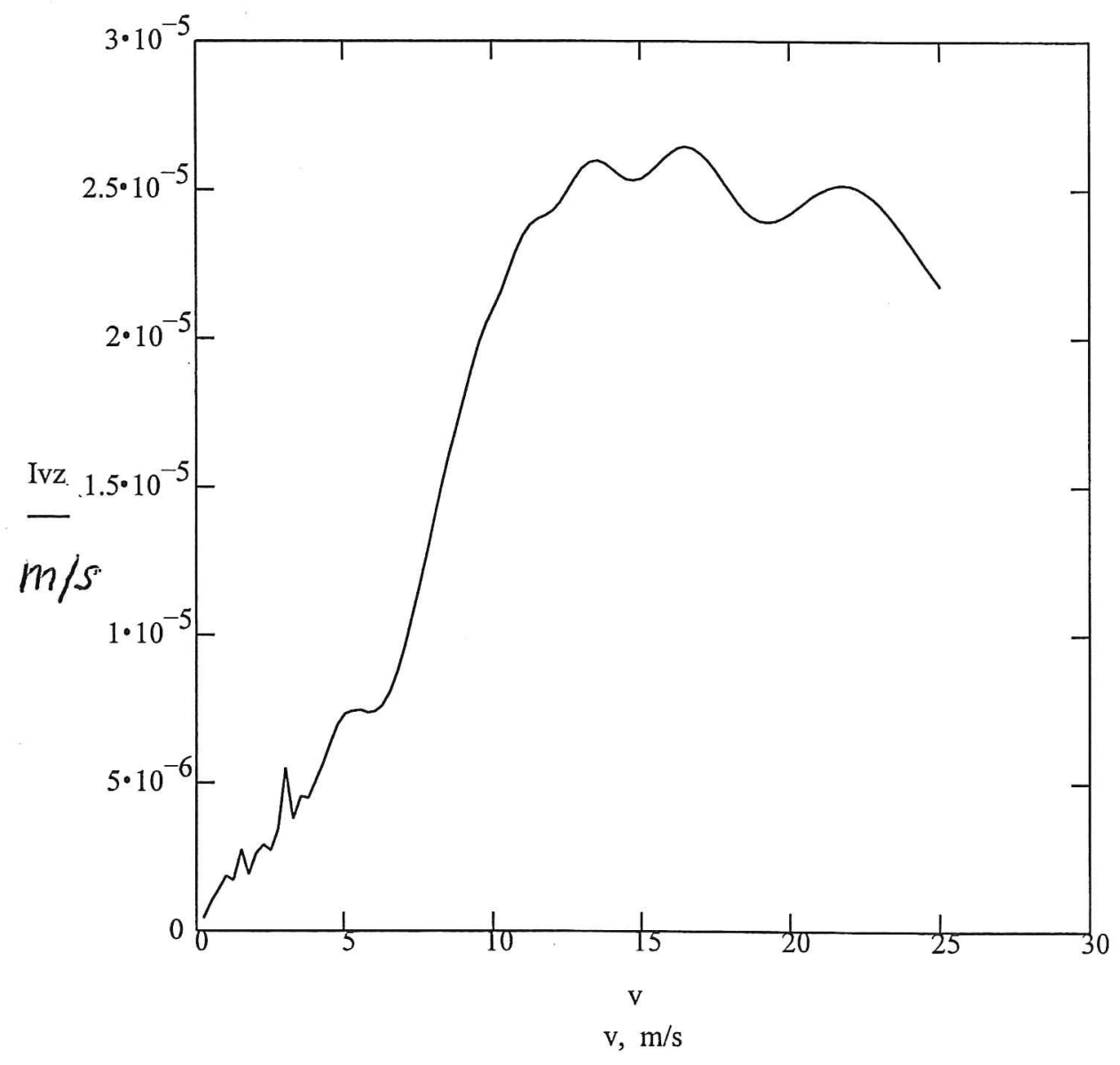

Fig. 6 


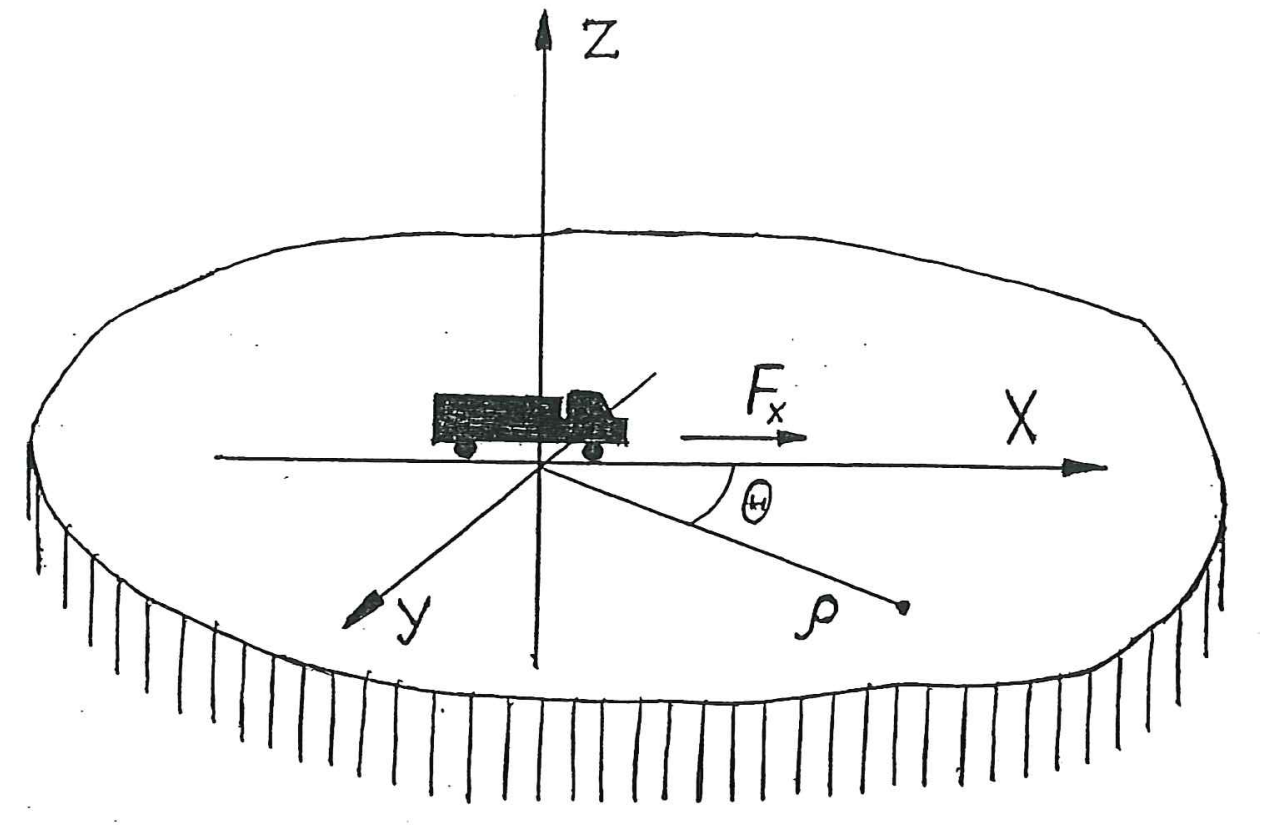

Fig. 7 


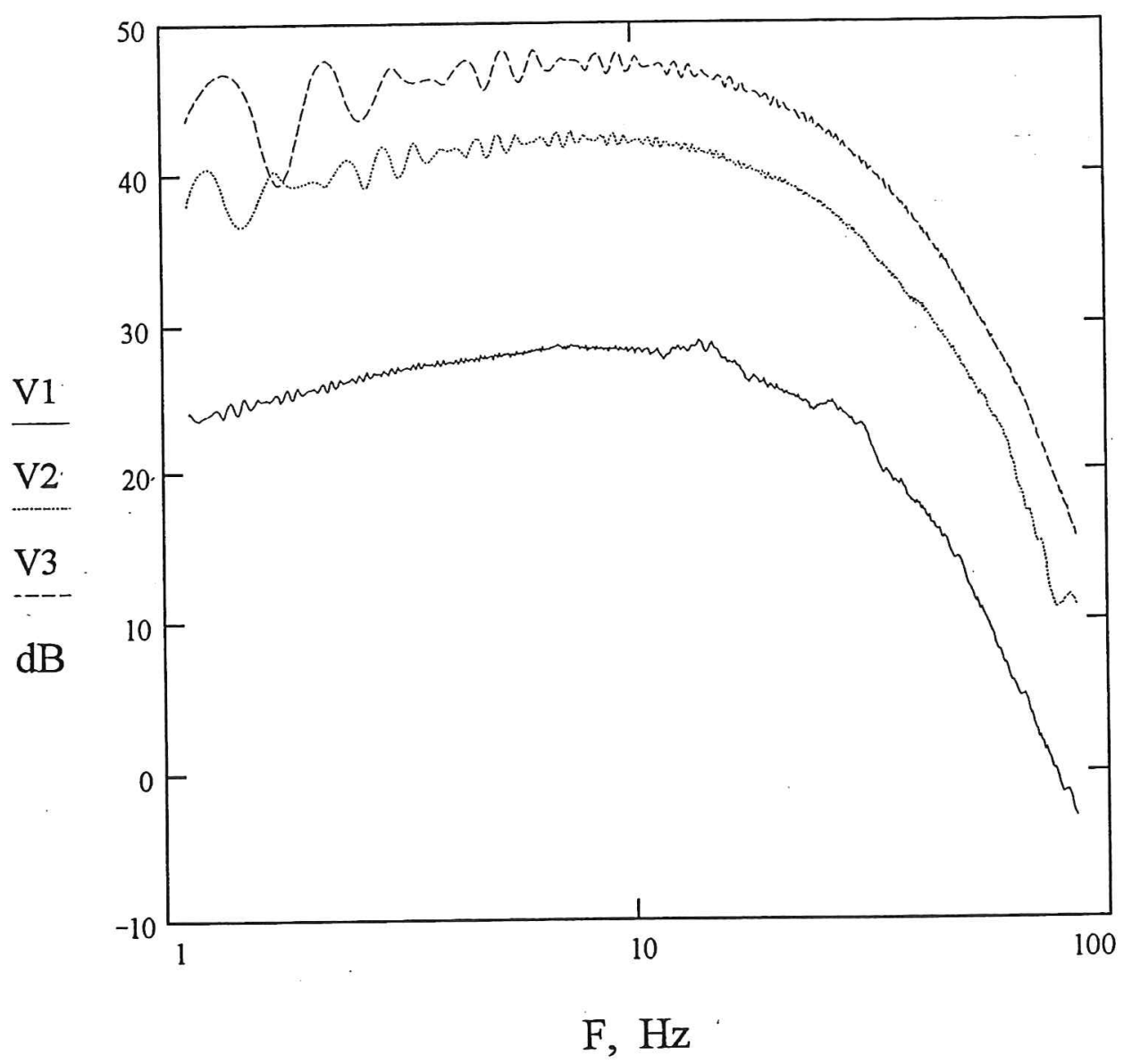

Fig. 8 


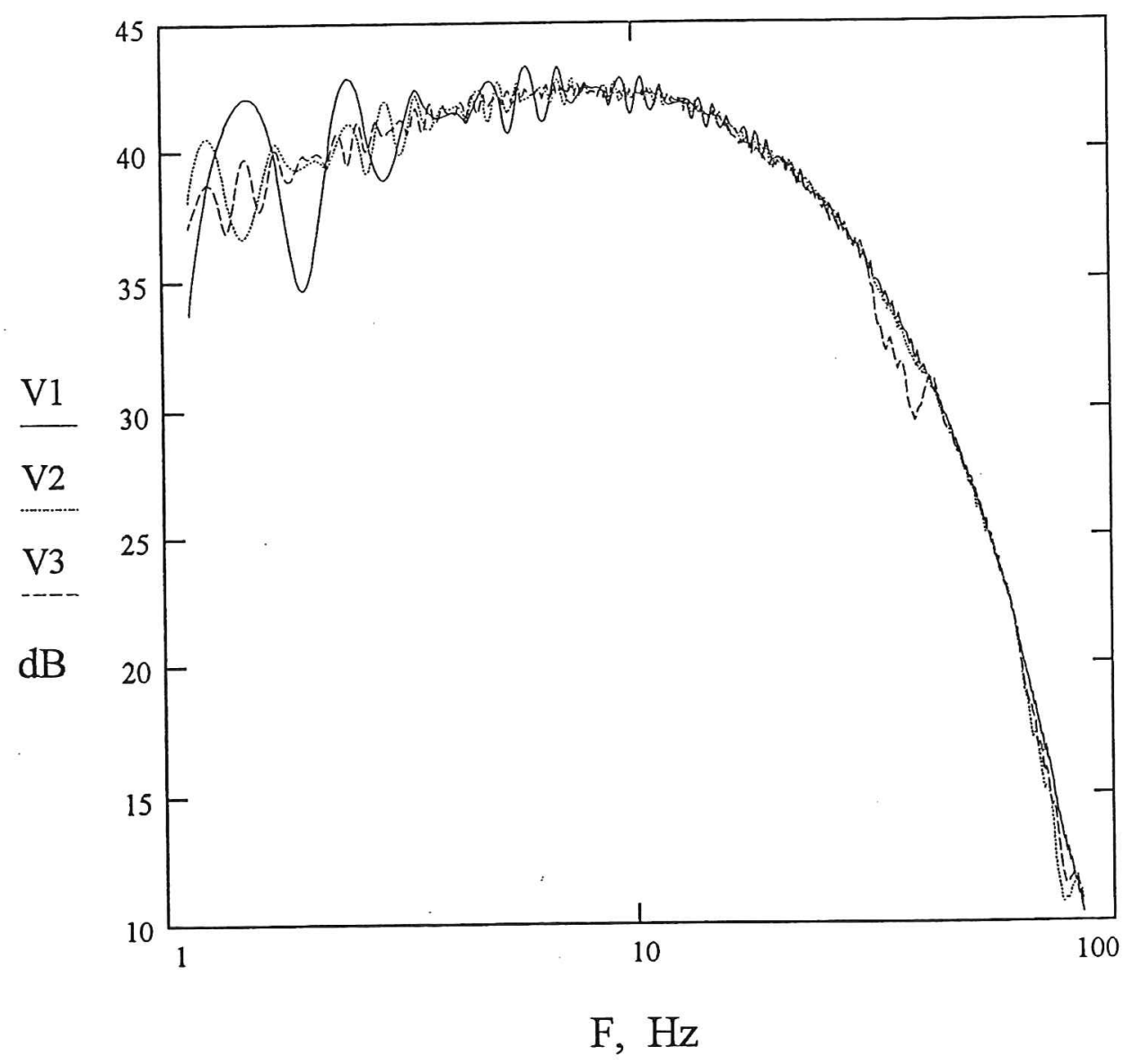

Fig. 9 


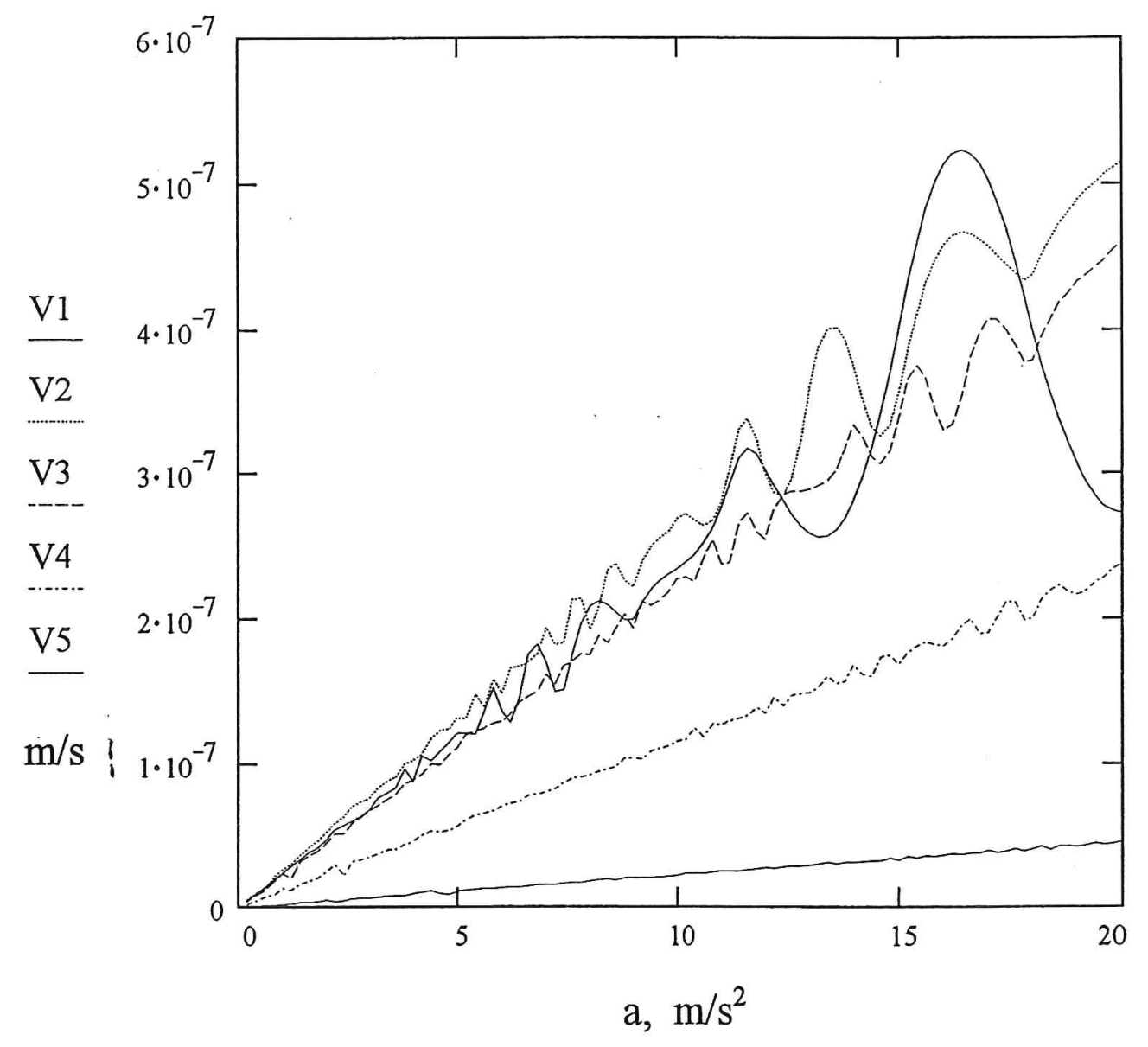

Fig. 10 


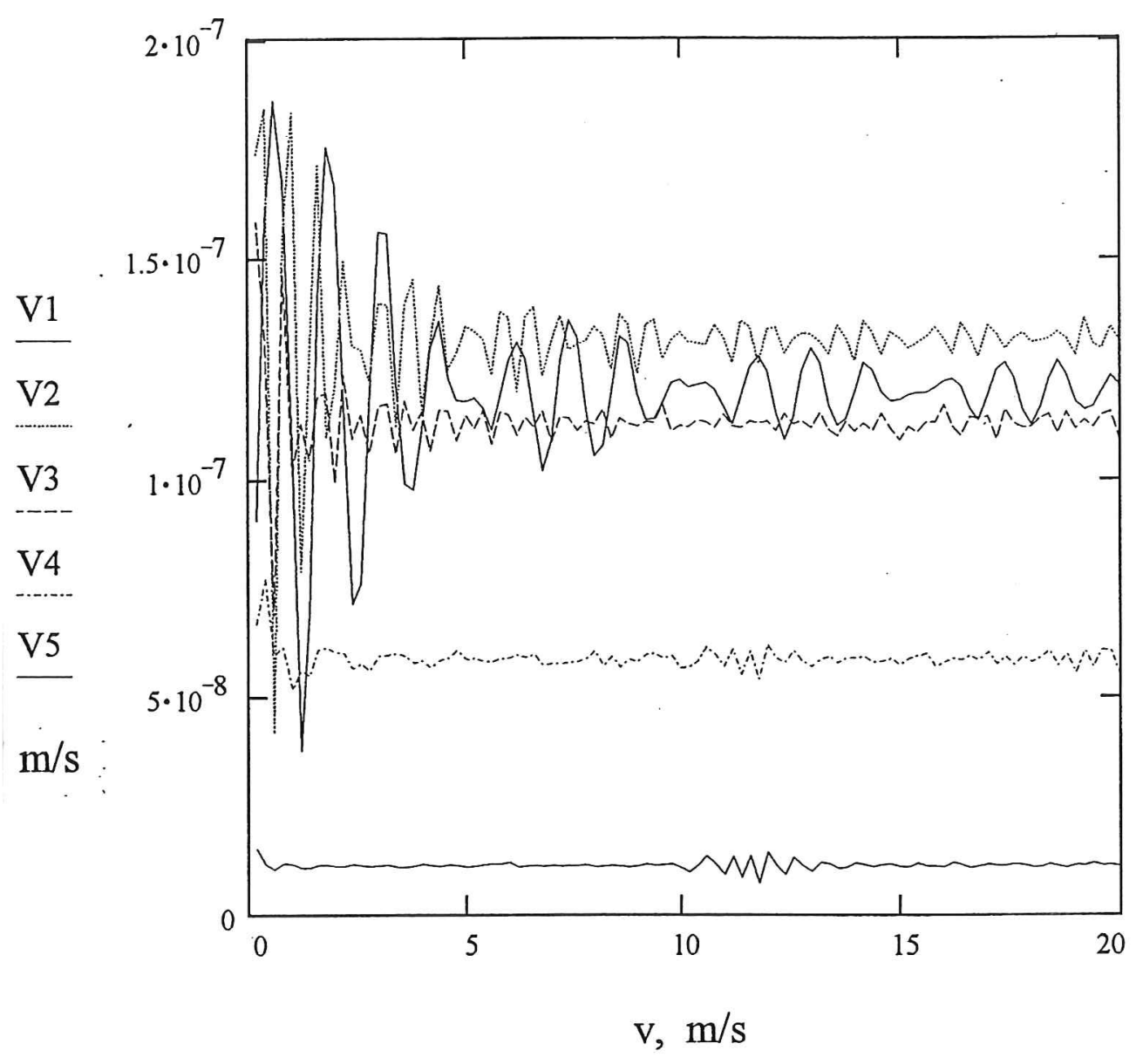

Fig. 11 